\title{
Changes in Healthcare Workers' Anxiety During Two Time Points of the COVID-19 Pandemic: Evidence From a Longitudinal Study
}

\author{
Lorena Cecilia López Steinmetz ${ }^{1,2}$ (D) Carla Romina Herrera ${ }^{3}$ · Shao Bing Fong ${ }^{4}$. \\ Juan Carlos Godoy ${ }^{1}$
}

Accepted: 27 September 2021

(c) The Author(s), under exclusive licence to Springer Science+Business Media, LLC, part of Springer Nature 2021

\begin{abstract}
Evidence on the within-person changes of healthcare workers' mental health across waves of COVID-19 cases during this pandemic is absent. The aim of this study is to examine the within-person changes of anxiety in Argentinean healthcare workers, adjusting for main demographic factors, region of residence, mental disorder history, and COVID-19 contagion, during the COVID-19 pandemic. A longitudinal web survey $(N=305)$ was conducted during two time points of the pandemic, one of which was an infection peak. Anxiety significantly increased across time. However, there were significant interaction effects modulating anxiety levels. The largest anxiety increases occurred in healthcare workers who were not sure if they had contracted COVID-19 while symptomatic. Irrespective of the time point, anxiety was the highest in healthcare workers from a region inside the country who were not sure if they had contracted COVID-19, either asymptomatic or symptomatic. An interaction effect between the mental disorder history and the COVID-19 contagion suggested that the anxiety outcomes were mainly due to the concern about the COVID-19 contagion, rather than due to pre-existing mental health vulnerabilities. Regardless of the starting point in anxiety levels, an increasing anxiety outcome may be expected among healthcare workers as the pandemic progresses. The uncertainty regarding COVID-19 contagion is a preventable and modifiable interacting factor to produce the worst anxiety outcomes among healthcare workers.
\end{abstract}

Keywords COVID-19 $\cdot$ Health personnel $\cdot$ Uncertainty $\cdot$ Infections, Coronavirus $\cdot$ Mental disorders $\cdot$ Developing countries

Lorena Cecilia López Steinmetz

cecilialopezsteinmetz@unc.edu.ar

1 Laboratorio de Psicología, Instituto de Investigaciones Psicológicas (IIPsi), Facultad de Psicología, Universidad Nacional de Córdoba (UNC) - Consejo Nacional de Investigaciones Científicas y Técnicas (CONICET), Boulevard de La Reforma Esquina Enfermera Gordillo, s/n, 2do piso, Ciudad Universitaria, Córdoba, Argentina

2 Universidad Siglo 21, Córdoba, Argentina

3 Servicio de Guardia, Hospital Wenceslao Gallardo, Jujuy, Argentina

$4 \quad$ Université de Rennes 1, Rennes, France 


\section{Introduction}

The coronavirus disease 2019 (COVID-19) that started as an outbreak in China in late December 2019 has rapidly scaled to an unprecedented pandemic. The unpreparedness of healthcare systems to tackle this sudden pandemic has contributed to the rising number of cases and, subsequently, to the job overload in healthcare workers.

Adverse mental health outcomes among healthcare workers have emerged during previous disease outbreaks (Liu et al., 2012; Maunder et al., 2003, 2006). Likewise, such outcomes have been reported by cross-sectional studies during the COVID-19 pandemic (Pappa et al., 2020; Spoorthy et al., 2020). However, longitudinal evidence is lacking. To date, there is only one published peer-reviewed longitudinal study assessing psychological problems in healthcare workers (Cai et al., 2020), but it is based exclusively in nurses and failed to assess the within-person changes on the mental health outcomes, which is a key information.

It has been widely recognized that healthcare workers are required to work under extreme pressure during this pandemic, and thus, negative mental health outcomes are expected to occur among them as a consequence of the COVID-19 pandemic (Gold, 2020; Greenberg et al., 2020; Kristal \& McNeil, 2020; Walton et al., 2020). Nevertheless, evidence on the within-person changes of healthcare workers' mental health across waves of COVID-19 cases during this pandemic is absent. Our study is a first step in addressing this gap.

In this study, we hypothesized that the levels of anxiety would be higher in healthcare workers from a region with the highest rates of COVID-19 cases as compared to those from a region with the lowest rates of these cases. In addition, we hypothesized that the levels of anxiety in healthcare workers will increase as the pandemic progresses. The point at issue questions how high will anxiety levels be and which are the main predictors associated with such increase. Thus, the main aim of this study is to examine the within-person changes in the levels of anxiety in Argentinean healthcare workers, adjusting for main demographic factors, region, mental disorder history, and COVID-19 contagion, during two time points of the COVID-19 pandemic.

\section{Method}

\section{Design}

This study is part of a broader research, in which we used a longitudinal design with two repeated-measures for assessing anxiety-state, general discomfort and psychological distress. In this paper, we focused only on anxiety outcomes. Sampling was one of convenience. The inclusion criteria were being a healthcare worker (irrespective of their training, e.g., physicians, nurses, psychologists, technicians, cleaners, administrative staff, or any other profession) and working during the COVID-19 pandemic in health institutions, public or private, from the Argentinean provinces of Buenos Aires, a metropolitan region that had up to 5,638 COVID-19 cases during the first measurement, or Jujuy, a region inside the country that had only up to 6 COVID-19 cases during the first measurement. Data collection for the first measurement extended from April 2 to May 30, 2020. The follow-up was carried out from September 15 to 30,2020 . The outcome variable was anxiety-state. The 
predictors that we analyzed were age, sex, region, mental disorder history, and COVID-19 contagion. In addition, we have analyzed the percentages of healthcare workers scoring as high anxiety-state in both measurements.

\section{Procedure}

Collection procedure was carried out online via the LimeSurvey software (UNC official license). We disseminated the invitations to participate, which included the link for the online survey, through e-mails, WhatsApp, and various social networks. No personal identification data was asked of participants during the survey, except for an email address and a cellphone number that was required for the follow-up. During September 15 to 30, 2020, participants were contacted to answer the online survey for the second time. In both the first and the second measurements, upon accessing the survey, participants were initially presented with the information sheet and informed consent form. Written informed consent was obtained from all subjects.

\section{Ethics}

This study was approved by the Ethics Committee of the Institute of Psychological Research, Faculty of Psychology, National University of Córdoba (CEIIPsi-UNC-CONICET; comite.etica.iipsi@psicologia.unc.edu.ar) on April 2, 2020.

\section{Variables and Instruments}

\section{Anxiety-State}

We used the 20-items subscale for anxiety-state of the State-Trait Anxiety Inventory (Spielberger et al., 1983) in its Spanish version (Cronbach's alpha=0.98; Ortuño Sierra et al., 2016), which evaluates anxiety as a transient emotional condition. The range of scores is between 0 and 60 . Higher scores indicate higher anxiety, but there are no standardized cutoff scores for this tool. Thus, we build up an ad hoc cutoff of $>30$ for classifying anxiety as high and $\leq 30$ for low anxiety, which was based on the middle point of range scores and the mean for the entire sample in the two measurements $\left(\operatorname{Mean}_{1 \mathrm{st}}\right.$ measurement $=31.97$, Mean $\left._{\text {follow-up }}=34.41\right)$. Hereinafter, we name anxiety-state as anxiety.

\section{Age}

We asked for the participants' age, by using a single item which allowed numerical responses. For data analysis, we grouped age into two ad hoc broad categories: Younger $(<40$ years old) and $\operatorname{Older}(\geq 40$ years old).

\section{Sex}

We asked for the participants' biological sex: Man or Woman. 


\section{Region}

We asked for the participants' site of residence. Since residing in Jujuy or Buenos Aires was explicated as an inclusion criterion, the answer options were Jujuy, Buenos Aires, and Other. Those choosing the Other option were then excluded. For data analysis, we used the categories inside the country and metropolitan region, corresponding to Jujuy and Buenos Aires, respectively.

\section{Mental Disorder History}

We used a single item to ask participants if they have ever been diagnosed with a mental problem (e.g., depression, anxiety, obsession, or any other). Answer options were dichotomous: No (absence) or Yes (presence).

\section{COVID-19 Contagion}

In the follow-up survey, we asked participants if they were infected with the COVID-19. The answer options were: No, I was not infected with the COVID-19; I don't know if I was infected with the COVID-19 and I have no symptoms of the disease; I don't know if I was infected with the COVID-19, but I have symptoms of the disease; Yes, I got sick with the COVID-19.

\section{Statistical Analyses}

All data was analyzed in RStudio version 3.6.2 (R Core Team, 2020). Reproducible code can be found at an online repository (López-Steinmetz, 2020). The level of significance was set at $p \leq 0.05$. Exact $p$-values were reported, except for $p$-values under 0.001 , which were reported as $p<0.001$. The distribution of the outcome variable was in the range of acceptable values for skewness and kurtosis ( -1 to 1 and -3 to 3 , respectively) (Brown, 2006); thus, parametric statistics were used. There were no missing data to handle.

We provided descriptive measures (percentages, mean, and standard deviation). Likewise, we applied the Student's $t$ test, the 2-sample test for equality of proportions, and the Pearson's r correlation coefficient, to test the first hypothesis of this study.

To address the second hypothesis and, thus, the main aim of this research, we used the multilevel approach rather than the repeated measures ANOVA due to two main reasons. First, we want to test the need to analyze nested models by regions, and second, we want to test for non-orthogonal contrasts. We ran mixed effects modeling by means of the multilevel approach for anxiety as the outcome variable. We analyzed models including the within-person factor (anxiety scores in the first measurement and the follow-up nested within participants) and the following between-group factors: age (younger, older; dummy coded as 0 and 1, respectively), sex (man, woman; dummy coded as 0 and 1 , respectively), region (inside the country, metropolitan region; dummy coded as 0 and 1 , respectively), mental disorder history (absence, presence; dummy coded as 0 and 1, respectively), and COVID-19 contagion (no, does not know and has no symptoms, does not know but has symptoms, yes). We have set non-orthogonal contrasts for this last predictor. We established the condition no (i.e., not having got 
infected with the COVID-19) as the baseline (dummy coded as 0 in all the contrasts). The contrasts compared the baseline versus each one of the remaining conditions of the COVID-19 contagion.

The first model only contained the intercept. We have built up the models by adding one predictor at a time in order to test the overall main effect of each predictor, and then, we built up the models to test all the possible interactions for two-predictor combinations. For the analysis of mixed effects modeling, we have used the nlme package (Pinheiro et al., 2020) with the maximum likelihood method. We have compared the fit of the models looking at two criteria: The Akaike information criterion (AIC) and the Log-Likelihood (logLik). For meaningful predictors in the best fitting model, we have calculated effect sizes (ES) by using the DSUR.noof package (Aufheimer, 2021).

\section{Results}

\section{Participants}

Three hundred thirty-nine healthcare workers participated in the first survey. The attrition was at $10.03 \%$ during the follow-up. Levels of anxiety at the first measurement did not significantly differ $\left(\mathrm{t}_{(39.82)}=-1.61, p=0.11,95 \% \mathrm{CI}=-7.82\right.$ to 0.88$)$ between healthcare workers who participated only in the first measurement $\left(\mathrm{Mean}_{\text {anxiety }}=28.50\right)$ and those who participated in both measurements $\left(\mathrm{Mean}_{\text {anxiety }}=31.97\right)$. In this work, we analyzed only the sample of 305 healthcare workers that have completed the online survey for the two-repeated measures. The $75.41 \%$ of the sample worked in one $(50 \%)$ or more $(50 \%)$ services implying in-person patient care: emergency ward (113), inpatient settings (81), ambulance (33), and/or outpatient consultations (124). The remaining

Table 1 Characteristics of the sample $(N=305$ healthcare workers)

\begin{tabular}{ll}
\hline Characteristics & $N(\%)$ \\
\hline Age & \\
$\quad$ Younger $(<40)$ & $150(49.18)$ \\
Older $(\geq 40)$ & $155(50.82)$ \\
Sex & \\
$\quad$ Man & $63(20.66)$ \\
$\quad$ Woman & $242(79.34)$ \\
Region & \\
Inside the country & $168(55.08)$ \\
Metropolitan & $137(44.92)$ \\
Mental disorder history & \\
Absence & $262(85.90)$ \\
Presence & $43(14.10)$ \\
COVID-19 contagion (during the follow-up) & \\
No & $138(45.25)$ \\
Does not know and has no symptoms & $99(32.46)$ \\
Does not know, but has symptoms & $20(6.56)$ \\
Yes & $48(15.74)$ \\
\hline
\end{tabular}




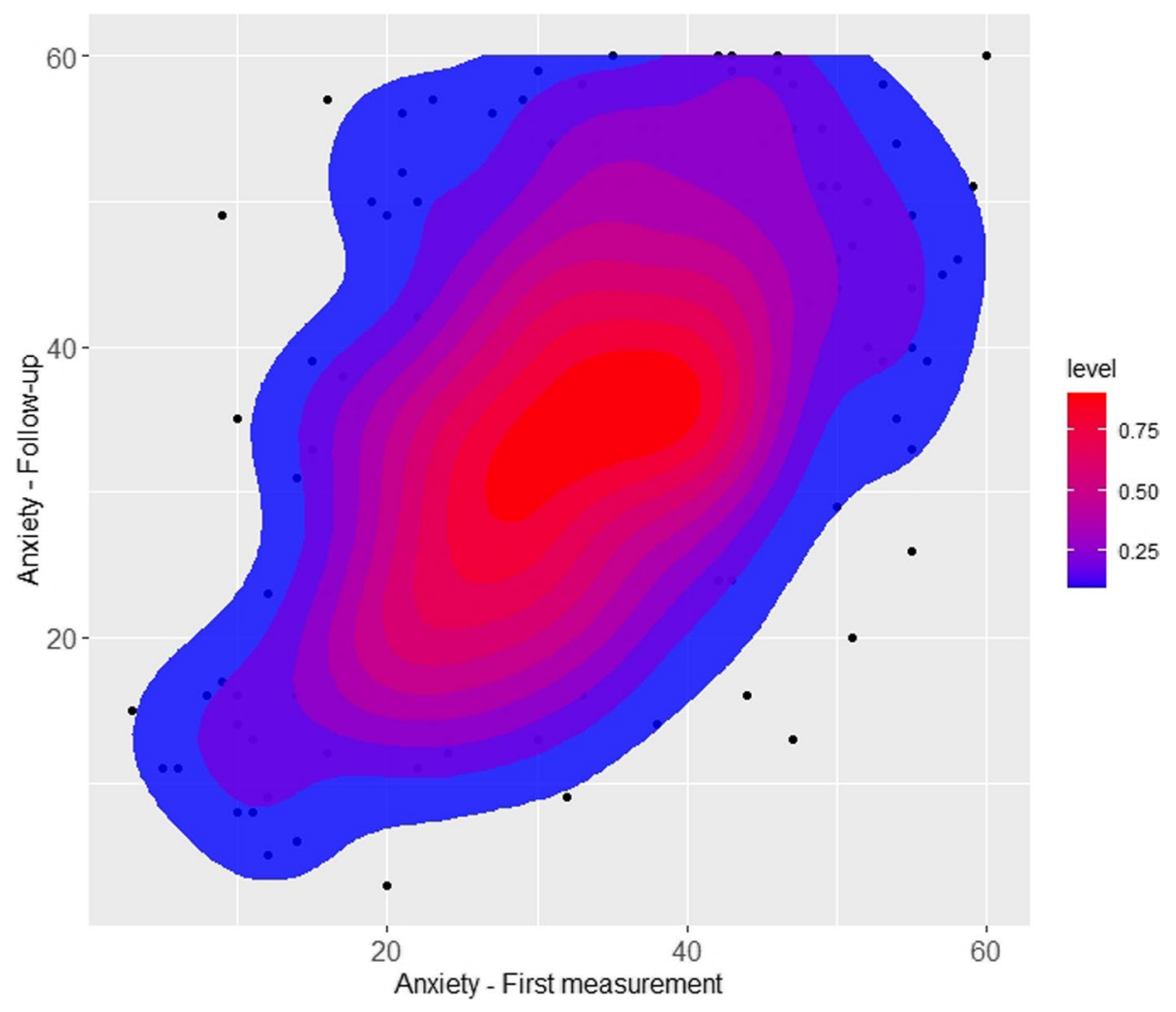

Note: Redder shades represent a higher density of cases and bluer shades represent a lower density of cases.

Fig. 1 Anxiety-state during the first measurement and the follow-up

$24.59 \%$ was committed to providing remote patient care. The remaining sample characteristics are summarized in Table 1.

\section{Anxiety Levels}

In the entire sample, $56.07 \%$ of healthcare workers scored as high anxiety in the first measurement, and significantly increased to $65.57 \%$ in the follow-up $\left(X^{2}{ }_{(1)}=5.79\right.$, $p=0.02,95 \% \mathrm{CI}=-0.17$ to -0.02 ). There was a positive and significant relationship between the levels of anxiety in the two measurements $(r=0.49, p<0.001$; Fig. 1). The mean level of anxiety in the first measurement was $31.97( \pm 11.28)$, while in the followup, the mean significantly increased to $34.41( \pm 12.97)\left(\mathrm{t}_{(304)}=-3.45, p<0.001\right)$.

Mean levels of anxiety during each measurement, grouped by age, sex, region, mental disorder history, and COVID-19 contagion, are summarized in Table 2. Regarding differences by regions, in the first measurement, the level of anxiety was lower in healthcare workers from inside the country region than those from the metropolitan region 
Table 2 Mean scores of anxiety in healthcare workers during the two-repeated measures $(N=305)$

\begin{tabular}{lll}
\hline Characteristics & Anxiety & \\
\cline { 2 - 3 } & $\begin{array}{l}\text { 1st measurement } \\
\text { M }( \pm \text { s.d. })\end{array}$ & $\begin{array}{l}\text { Follow-up } \\
\text { M }( \pm \text { s.d. })\end{array}$ \\
\hline Age & & \\
$\quad$ Younger $(<40)$ & $34.30( \pm 10.67)$ & $36.21( \pm 11.83)$ \\
$\quad$ Older $(\geq 40)$ & $29.72( \pm 11.43)$ & $32.66( \pm 13.80)$ \\
Sex & & \\
$\quad$ Man & $29.00( \pm 11.64)$ & $31.90( \pm 13.61)$ \\
$\quad$ Woman & $32.74( \pm 11.08)$ & $35.06( \pm 12.74)$ \\
Region & & \\
$\quad$ Inside the country & $30.61( \pm 11.93)$ & $33.40( \pm 15.05)$ \\
$\quad$ Metropolitan & $33.63( \pm 10.24)$ & $35.64( \pm 9.74)$ \\
Mental disorder history & & $33.47( \pm 12.98)$ \\
$\quad$ Absence & $31.24( \pm 11.50)$ & $40.12( \pm 11.45)$ \\
$\quad$ Presence & $36.44( \pm 8.73)$ & \\
COVID-19 contagion (during the follow-up $)$ & & $32.83( \pm 13.31)$ \\
$\quad$ No & $31.36( \pm 11.83)$ & $36.64( \pm 11.79)$ \\
$\quad$ Does not know and has no symptoms & $32.52( \pm 11.10)$ & $43.05( \pm 13.10)$ \\
$\quad$ Does not know, but has symptoms & $35.60( \pm 10.30)$ & $30.73( \pm 12.18)$ \\
$\quad$ Yes & $31.06( \pm 10.38)$ &
\end{tabular}

M, mean; \pm s.d., standard deviation

$\left(\mathrm{t}_{(302.17)}=-2.38, p=0.02\right)$, while in the follow-up, there was no statistically significant difference among them $\left(\mathrm{t}_{(288.95)}=-1.57, p=0.12\right)$.

\section{Within-Person Changes in Anxiety: Mixed Effects Modeling}

We evaluated the need to analyze nested models by regions, but allowing the intercepts to vary across regions did not significantly improve the model fit $\left(\mathrm{AIC}_{\text {start }}=2431.63\right.$, $\mathrm{AIC}_{\text {final }}=2433.62, p=0.90$; Fig. 2). Thus, the models that we have built up included only fixed effects. We found a significant main effect of the age $\left(X^{2}{ }_{(6)}=11.76, p<0.001\right.$; $\mathrm{AIC}=4694.24, \operatorname{logLik}=-2341.12)$ on anxiety. The levels of anxiety were higher in the younger group $(<40$ years old $)$, although the effect size was small $(\mathrm{ES}=0.16)$. The sex also had a main effect $\left(X^{2}{ }_{(7)}=4.69, p=0.03 ; \mathrm{AIC}=4691.56\right.$, logLik $\left.=-2338.78\right)$ on anxiety, but in the final model, it does not remain as meaningful $\left(b=1.70, t_{(279)}=0.56, p=0.57\right)$. There were also main effects of time $\left(X_{(5)}^{2}=11.74, p<0.001\right.$; AIC $=4704.00$, logLik $=-2347.00)$, the mental disorder history $\left(X^{2}{ }_{(9)}=15.77, p<0.001\right.$; AIC $=4677.70$, logLik $=-2329.85)$, and the COVID-19 contagion $\left(X_{(12)}^{2}=11.77, p=0.01 ; \mathrm{AIC}=4671.93\right.$, $\operatorname{logLik}=-2323.96)$ on anxiety. However, there were significant interactions involving these variables, which supersede it; thus, these effects should not be interpreted as main effects. On the other hand, the levels of anxiety were similar between the regions $\left(X_{(8)}^{2}=2.09, p=0.15 ; \mathrm{AIC}=4691.47, \log \mathrm{Lik}=-2337.73\right)$.

There was a significant interaction effect between time (the within-person factor) and the COVID-19 contagion $\left(X_{(19)}^{2}=9.07, p=0.03\right.$; $\left.\mathrm{AIC}=4675.65, \log \mathrm{Lik}=-2318.82\right)$ on 


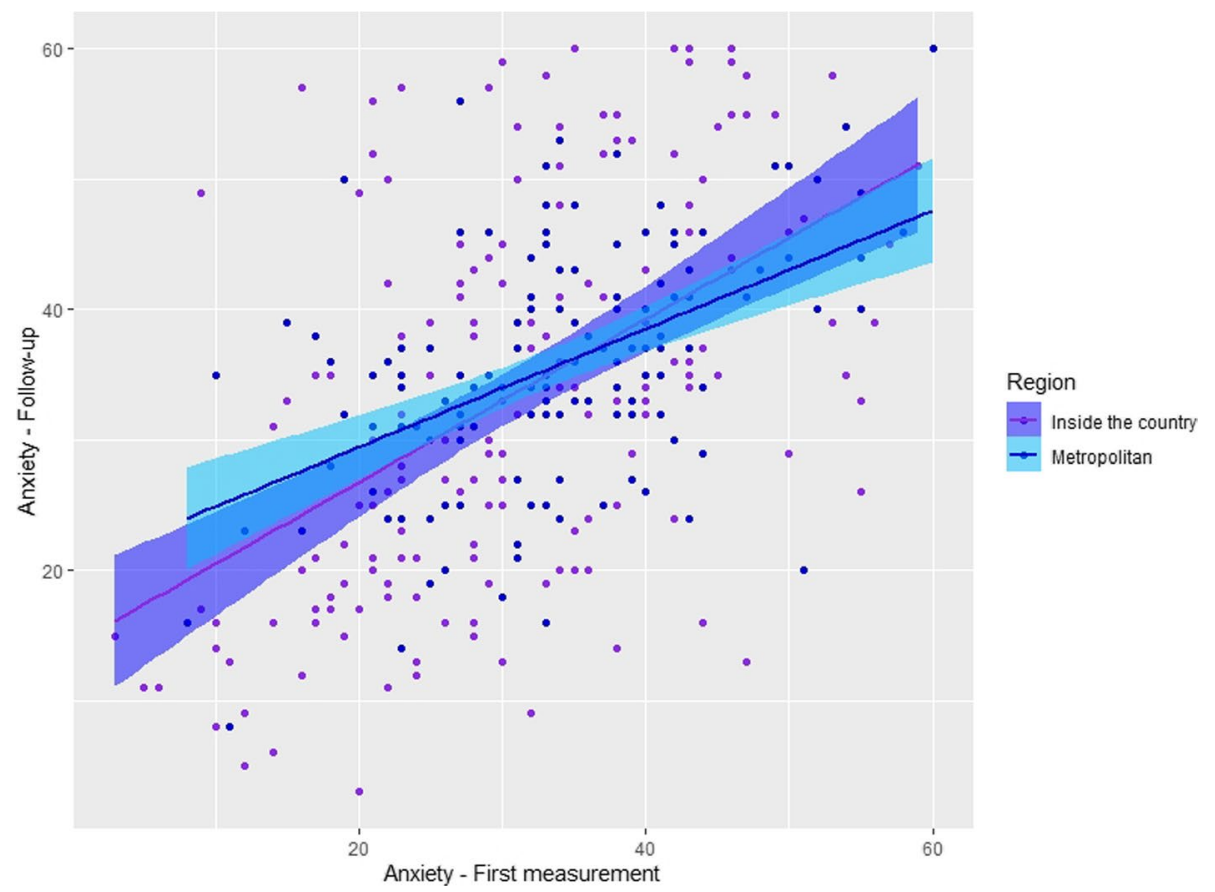

Fig. 2 Anxiety-state during the first measurement and the follow-up by regions

anxiety. The contrasts revealed that, compared to those who had not been infected with the COVID-19, anxiety significantly increased from the first measurement to the follow-up in those who did not know if they had been infected with the COVID-19, but were symptomatic $\left(b=5.85, t_{(297)}=1.95, p=0.05, \mathrm{ES}=0.11\right)$. On the contrary, there were no significant differences in anxiety across time when comparing those who had not been infected with the COVID-19 to those who did not know if they had been infected with the COVID-19 and had no symptoms of the disease $\left(b=2.90, t_{(297)}=1.75, p=0.08\right)$, and to those who had gotten sick with COVID-19 $\left(b=-1.90, t_{(297)}=-0.91, p=0.37\right)$.

There was also a significant interaction effect between the region and the COVID-19 contagion $\left(X_{(34)}^{2}=12.87, p=0.005 ; \mathrm{AIC}=4682.50\right.$, logLik $\left.=-2307.25\right)$ on anxiety. Compared to those who had not been infected with the COVID-19, anxiety was significantly higher among those who did not know if they had been infected with the COVID-19, whether they were asymptomatic $\left(b=-5.93, t_{(279)}=-2.29, p=0.02, \mathrm{ES}=0.14\right)$ or symptomatic $\left(b=-10.24, t_{(279)}=-1.93, p=0.05, \mathrm{ES}=0.11\right)$, although only among healthcare workers from the region inside the country, but not among healthcare workers from the metropolitan region. On the contrary, there were no significant differences in anxiety when comparing those who had not been infected with the COVID-19 to those who had gotten sick with COVID-19, among healthcare workers from both regions $\left(b=3.90, t_{(279)}=1.05\right.$, $p=0.29$ ).

Finally, there was also a significant interaction effect between the mental disorder history and the COVID-19 contagion $\left(X_{(37)}^{2}=10.03, p=0.02 ; \mathrm{AIC}=4678.47, \log \mathrm{Lik}=-2302.23\right)$ on anxiety. When comparing those who had not been infected with the COVID-19 to those who did not know if they had been infected with the COVID-19, but were symptomatic, anxiety was significantly higher among healthcare workers without mental disorder 
history than among their counterparts with such a background $\left(b=-19.45, t_{(279)}=-2.98\right.$, $p=0.003$, ES $=0.18$ ). Instead, there were no significant differences in anxiety influenced by the mental disorder history when comparing those who had not been infected with the COVID-19 to those who did not know if they had been infected with the COVID-19 and were asymptomatic $\left(b=-4.77, t_{(279)}=-1.33, p=0.19\right)$, and to those who had gotten sick with COVID-19 $\left(b=-1.26, t_{(279)}=-0.21, p=0.83\right)$.

The remaining interactions that we tested were not meaningful $(p>0.05$; data not shown). Table 3 summarizes the model best fitting the data on anxiety.

\section{Discussion}

This study is the first to track the within-person changes in the levels of anxiety in healthcare workers during two time points of the COVID-19 pandemic. This study was based on an Argentinean sample of healthcare workers, to analyze the changes in the anxiety outcomes, adjusting for main demographic factors, region, and some relevant health-related factors.

Consistent with our first hypothesis, we found higher levels of anxiety among healthcare workers from the metropolitan region compared to those from the region inside the country at the first time point of this study, when there were more than five thousand of COVID-19 cases in the former region and almost no cases in the latter region. Nonetheless, these differences disappeared in the follow-up, when the pandemic has progressed. By then, the metropolitan region reached 417,677 COVID-19 cases and the region inside the country reached 15,670 cases (Ministry of Health, 2020). This meant around 2610 and 2328 COVID-19 cases per 100,000 inhabitants in the former and the latter regions, respectively. Thus, such numbers of COVID-19 cases in both regions imply a worrying worsening of the health situation and a pressing workload for healthcare workers, which could result in higher burden of negative mental health outcomes such as anxiety. Indeed, as it was expected according to our second hypothesis, we found increasing levels of anxiety in healthcare workers from the first time point to the follow-up. Overall, these findings suggest that irrespective of the starting point in anxiety levels among healthcare workers, an increasing anxiety outcome may be expected among them as the pandemic progresses.

Furthermore, beyond the negative impact that implies for healthcare workers an increasing curve of the pandemic itself (e.g., overwhelming workload, working under extreme pressures, depletion of personal protective equipment, covering additional shifts, among others) (Greenberg et al., 2020; Lai et al., 2020), our findings warn that the increase of anxiety among healthcare workers is moderated by the concern regarding the COVID-19 contagion. Both negative mental health outcomes (i.e., anxiety) and relevant predictors for such outcomes (i.e., COVID-19 contagion) had been suspected to occur during the current pandemic based on previous epidemic and disease outbreaks (Carmassi et al., 2020) and also based on current but cross-sectional evidence (Muller et al., 2020; Pappa et al., 2020; Spoorthy et al., 2020), though they had not been supported with longitudinal evidence during the COVID-19 pandemic, until now. Published peer reviewed longitudinal studies on mental health outcomes in healthcare workers during the COVID-19 pandemic are lacking and our study is a first step in order to fill this gap.

As it was suggested by the review of Carmassi et al. (2020), based on previous crosssectional and some longitudinal evidence from the Severe Acute Respiratory Syndrome (SARS) and Middle East Respiratory Syndrome (MERS) outbreaks, it may be expected to 


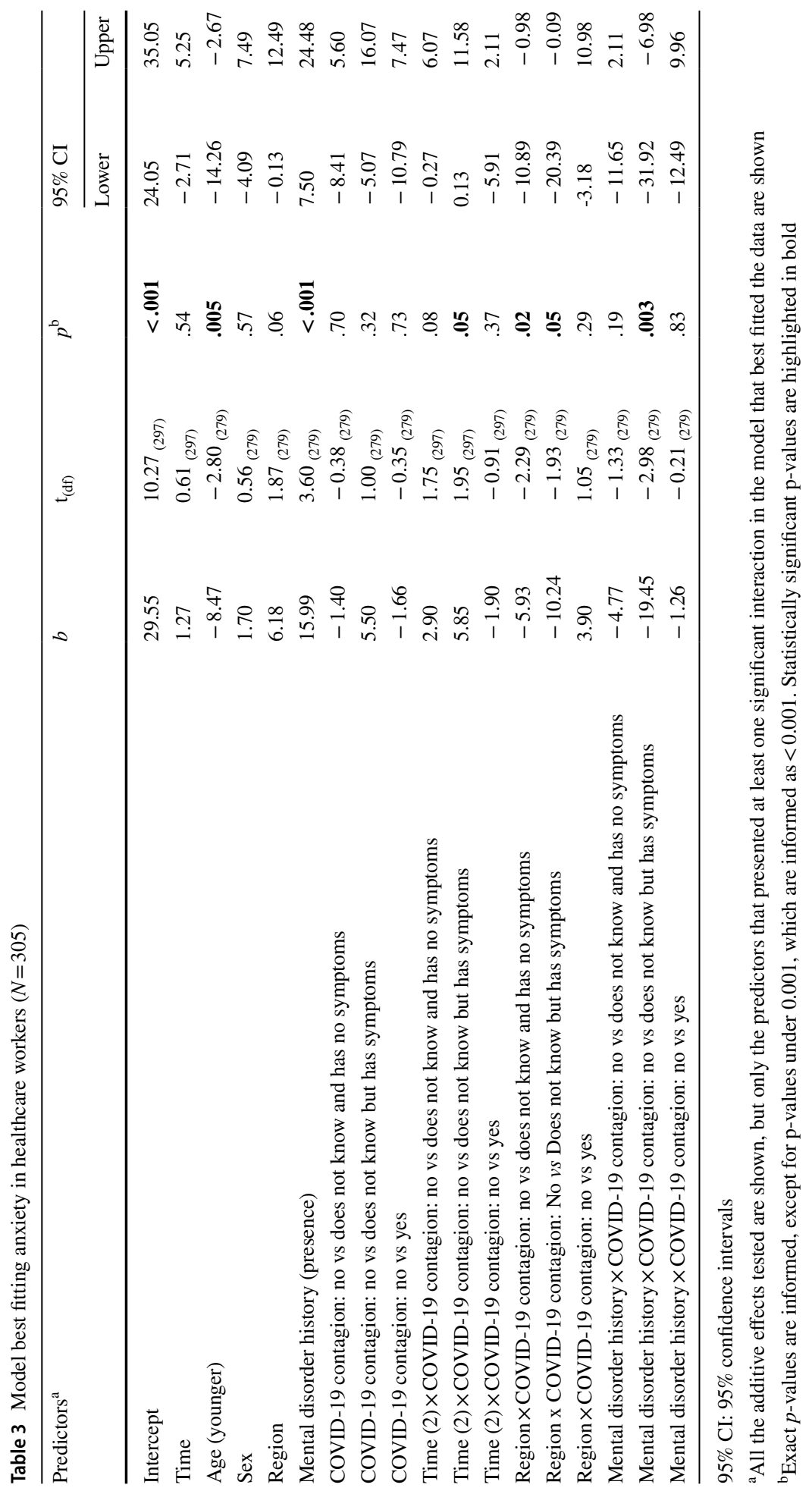


find the worst mental health outcomes among healthcare workers who had gotten sick with the COVID-19. Nonetheless, based on our current longitudinal study, the largest increases in anxiety levels are not among healthcare workers who have been gotten sick, but in those who wondered if they had been infected with the COVID-19, while being symptomatic. Considering that majority of the healthcare workers in our study were involved in direct patient care, these findings suggest that the frequent exposure to the COVID-19 in their working environment contributes to the uncertainty and suspicion about the contagion, which becomes a heavier factor for the increase of anxiety among them than the actual contagion. Similar hints have emerged with general discomfort and psychological distress outcomes among healthcare workers during the current pandemic (López Steinmetz et al., 2021). During the SARS outbreak, the perception of personal danger, for instance related to shortages of hospital masks, was exacerbated by the uncertainty in healthcare workers (Maunder et al., 2003). Moreover, uncertainty, although not referring to the COVID-19 contagion, has been demonstrated as related to negative mental health outcomes, namely anxiety and depression, among the general population both prior (Carleton et al., 2012) and during (Rettie \& Daniels, 2020) the COVID-19 pandemic. Leading with uncertainty is a routine part of the medical practice (Eddy, 1984) and, in a more general sense, is part of the work in all healthcare-related professions. However, this known uncertainty is usually referring to aspects pertaining to the patient (e.g., her/his diagnosis, treatment, etc.) or to the disease (e.g., its etiology, prognosis, etc.), but not to the health state of the healthcare workers themselves, like in the unprecedented COVID-19 pandemic. This last kind of uncertainty, commonly unknown to most healthcare workers, often emerges during contagious disease outbreaks. In this sense, during viral epidemic outbreaks, a higher prevalence of a number of mental health problems, mainly anxiety and depression, was associated with a higher perception of threat and risk among healthcare workers (Serrano-Ripoll et al., 2020). Fortunately, the uncertainty regarding COVID-19 contagion in healthcare workers is both a preventable and a modifiable factor by means of providing adequate protection supplies and more COVID-19 tests for them.

Furthermore, the anxiety levels of healthcare workers were moderated by the interaction effect between the region and the COVID-19 contagion. We believe that additional variables not assessed in our study may help to interpret this finding, for instance, shortages in personal protective equipment, which is a major source of distress in healthcare workers during the COVID-19 pandemic (Shechter et al., 2020). Such shortages are known to be related to economic reasons and hurdles in transportation and distribution logistics, among other reasons. Although the shortage of personal protective equipment affected countries all around the world during this pandemic, this problem is two-fold for regions with lower income and with more serious distribution problems (Carter et al., 2020). In this regard, compared to the metropolitan region, the region inside the country is of a lower income and has remote access, since it is located in the north westernmost of Argentina. Thus, shortages in personal protective equipment may have affected healthcare institutions in this region-as it has been reported in the media (see e.g., Infobae, 2020)-more than institutions from the metropolitan region and such scarcity may have acted as an anxiogenic factor. Indeed, our results show that, irrespective of the time point, anxiety was the highest in healthcare workers from the region inside the country who wondered if they had been infected with the COVID-19, whether they were asymptomatic or symptomatic.

Finally, we have also found an interaction effect between the mental disorder history and the COVID-19 contagion on anxiety. Strikingly, the highest anxiety affected healthcare workers without mental disorder history who wondered if they had been infected with the COVID-19 and were symptomatic. This is contrary to what would be expected based on 
studies during the SARS outbreak, which highlighted a relationship between having a history of psychiatric disorders and worst mental health outcomes among healthcare workers (Lancee et al., 2008; Su et al., 2007). However, such outcomes would be, in turn, inversely associated with years of healthcare experience and the perceived adequacy of training and support (Lancee et al., 2008), variables that we have failed to assess in our study and may help to explain the opposite findings. Nonetheless, the cited studies are not entirely comparable to our study. For instance, they were based on smaller samples than ours, which were comprised of either 102 nurses solely (Su et al., 2007) or 139 healthcare workers (Lancee et al., 2008). In addition, the findings of the latter study correspond to a follow-up of 1 to 2 years after the SARS outbreak, rather than a follow-up during the sanitary event as in our study. All in all, the interaction effect between the mental disorder history and the COVID-19 contagion emerged in our study would suggest that the anxiety outcomes found in healthcare workers are mainly due to the concern about the COVID-19 contagion, rather than due to pre-existing mental health vulnerabilities.

\section{Limitations}

The findings of this study, although valuable, should be considered in the context of some limitations. First, as we discussed earlier in this paper, additional factors not measured in this study may be relevant to thoroughly understand the negative mental health impacts in healthcare workers during the COVID-19 pandemic and further research is needed to address such factors. Second, the sample was not representative of all Argentinean healthcare workers, though it included data of healthcare workers from well-balanced metropolitan and non-metropolitan or rural areas and there was a low attrition between the two measurements. Third, most women participated. Although to some extent this reflects the fact that women are majoritarian among Argentinean healthcare workers (PNUD, 2018), the uneven sex distribution of the sample is a potential bias source. Fourth, we failed to measure anxiety prior to the COVID-19 pandemic; thus, we cannot assure that the outcome of interest was not present at the start of the study. However, the longitudinal design we used allowed us to ascertain that such outcome meaningfully increased as pandemic progresses, irrespective of the different starting anxiety level of healthcare workers from both regions.

\section{Implications}

Despite the abovementioned limitations, this study provides valuable longitudinal evidence-based knowledge on the within-person changes in the levels of anxiety of healthcare workers during the COVID-19 pandemic and identifies foremost factors conducive to anxiety outcomes. The uncertainty regarding the COVID-19 contagion, a preventable and modifiable factor interacting to produce the worst anxiety outcomes among healthcare workers, should be promptly addressed by public health officials and government officials, for instance, providing adequate protection supplies and more COVID-19 tests for healthcare workers may reduce anxiety levels among them.

Acknowledgements We thank the participants for taking the time to contribute to this study. We also wish to acknowledge all healthcare workers for their commitment with the care of patients, families, and our community during the COVID-19 pandemic. 
Data Availability The data that support the findings of this study and the reproducible $\mathrm{R}$ code for data analysis are available in the Open Science Framework (OSF) repository, https://doi.org/10.17605/OSF.IO/ VMEK2.

\section{Declarations}

Ethics Approval The protocol of this research was approved by the Ethics Committee of the Institute of Psychological Research, Faculty of Psychology, National University of Córdoba (CEIIPsi-UNC-CONICET; comite.etica.iipsi@psicologia.unc.edu.ar).

Consent to Participate Informed consent was obtained from all individual participants included in the study.

Conflict of Interest The authors declare no competing interests.

\section{References}

Aufheimer, M. (2021). DSUR.noof: Collection of Additional Functions Used in Andy Field's 'Discovering Statistics Using R'. R package version 0.1.1. http://github.com/Frostarella/DSUR.noof. Accessed 4 Oct 2021

Brown, T. A. (2006). Confirmatory factor analysis for applied research. Guilford Press.

Cai, Z., Cui, Q., Liu, Z., Li, J., Gong, X., Liu, J., Wan, Z., Yuan, X., Li, X., Chen, C., \& Wang, G. (2020). Nurses endured high risks of psychological problems under the epidemic of COVID-19 in a longitudinal study in Wuhan China. Journal of Psychiatric Research, 131, 132-137. https://doi.org/10.1016/j. jpsychires.2020.09.007

Carleton, R. N., Mulvogue, M. K., Thibodeau, M. A., McCabe, R. E., Antony, M. M., \& Asmundson, J. G. (2012). Increasingly certain about uncertainty: Intolerance of uncertainty across anxiety and depression. Journal of Affective Disorders, 26(3), 468-479. https://doi.org/10.1016/j.janxdis.2012.01.011

Carmassi, C., Foghi, C., Dell'Oste, V., Cordone, A., Bertelloni, C. A., Bui, E., \& Dell'Osso, L. (2020). PTSD symptoms in healthcare workers facing the three coronavirus outbreaks: What can we expect after the COVID-19 pandemic. Psychiatry Research, 292, 113312. https://doi.org/10.1016/j.psychres. 2020.113312

Carter, C., Anh, T. L., \& N., \& Notter, J. . (2020). COVID-19 disease: Perspectives in low- and middleincome countries. Clinics in Integrated Care, 1, 100005. https://doi.org/10.1016/j.intcar.2020.100005

Eddy, D. M. (1984). Variations in physician practice: The role of uncertainty. Health Affairs (millwood), 3(2), 74-89. https://doi.org/10.1377/hlthaff.3.2.74

Gold, J. A. (2020). Covid-19: Adverse mental health outcomes for healthcare workers. BMJ, 369, m1815. https://doi.org/10.1136/bmj.m1815

Greenberg, N., Docherty, M., Gnanapragasam, S., \& Wessely, S. (2020). Managing mental health challenges faced by healthcare workers during covid-19 pandemic. BMJ, 368, m1211. https://doi.org/10. 1136/bmj.m1211

Infobae. (2020, August 18). Una médica jujeña, al borde del llanto: "No hay camas, no hay oxígeno, estamos colapsados" [A doctor from Jujuy, on the verge of cryning: "No beds, no oxygen, we are collapsed”]. Infobae. https://www.infobae.com/sociedad/2020/08/18/una-medica-jujena-al-borde-del-1lanto-no-hay-camas-no-hay-oxigeno-estamos-colapsados/. Accessed 5 Sept 2021

Kristal, J. H., \& McNeil, R. L., Jr. (2020). Responding to the hidden pandemic for healthcare workers: Stress. Nature Medicine, 26(5), 639. https://doi.org/10.1038/s41591-020-0878-4

Lai, J., Ma, S., Wang, Y., Cai, Z., Hu, J., Wei, N., Wu, J., Du, H., Chen, T., Li, R., Tan, H., Kang, L., Yao, L., Huang, M., Wang, H., Wang, G., Liu, Z., \& Hu, S. (2020). Factors associated with mental health outcomes among health care workers exposed to coronavirus disease 2019. JAMA Network Open, 3(3), e203976. https://doi.org/10.1001/jamanetworkopen.2020.3976

Lancee, D. J., Maunder, R. G., Goldbloom, D. S., \& Coauthors for the Impact of SARS Study. (2008). Prevalence of psychiatric disorders among Toronto hospital workers one to two years after the SARS outbreak. Psychiatric Services, 59(1), 91-95. https://doi.org/10.1176/ps.2008.59.1.91

Liu, X., Kakade, M., Fuller, C. J., Fan, B., Fang, Y., Kong, J., Guan, Z., \& Wu, P. (2012). Depression after exposure to stressful events: Lessons learned from the severe acute respiratory syndrome epidemic. Comprehensive Psychiatry, 53(1), 15-23. https://doi.org/10.1016/j.comppsych.2011.02.003 
López Steinmetz, L. C. (2020). Dataset and R Code for: Changes in anxiety of healthcare workers during the COVID-19 pandemic: Evidence from a longitudinal study. Open Science Framework (OSF). 10.17605/OSF.IO/VMEK2

López Steinmetz, L. C., Herrera, C. R., Fong, S. B., \& Godoy, J. C. (2021). A longitudinal study on the changes in mental health of healthcare workers during the COVID-19 pandemic. Psychiatry: Interpersonal and Biological Processes. https://doi.org/10.1080/00332747.2021.1940469

Maunder, R., Hunter, J., Vincent, L., Bennett, J., Peladeau, N., Leszcz, M., Sadavoy, J., Verhaeghe, L. M., Steinberg, R., \& Mazzulli, T. (2003). The immediate psychological and occupational impact of the 2003 SARS outbreak in a teaching hospital. CMAJ: Canadian Medical Association Journal, 168(10), 1245-1251.

Maunder, R. G., Lancee, W. J., Balderson, K. E., Bennett, J. P., Borgundvaag, B., Evans, S., Fernandes, C. M., Goldbloom, D. S., Gupta, M., Hunter, J. J., McGillis Hall, L., Nagle, L. M., Pain, C., Peczeniuk, S. S., Raymond, G., Read, N., Rourke, S. B., Steinberg, R. J., Stewart, T. E., ... Wasylenki, D. A. (2006). Long-term psychological and occupational effects of providing hospital healthcare during SARS outbreak. Emerging Infectious Diseases, 12(12), 1924-1932. https://doi.org/10.3201/eid1212.060584

Ministry of Health (2020). COVID-19 daily report, $n^{o} 390$ [COVID-19 reporte diario, $n^{o} 390$ ]. https://www. argentina.gob.ar/sites/default/files/30-09-20-reporte-vespertino-covid-19.pdf. Accessed 30 Sept 2021

Muller, A. E., Hafstad, E. V., Himmels, J., Smedslund, G., Flottorp, S., Stensland, S. Ø., Stroobants, S., Van de Velde, S., \& Vist, G. E. (2020). The mental health impact of the covid-19 pandemic on healthcare workers, and interventions to help them: A rapid systematic review. Psychiatry Research, 293, 113441. https://doi.org/10.1016/j.psychres.2020.113441

Ortuño Sierra, J., García Velasco, L., Inchausti, F., Debbané, M., \& Fonseca Pedrero, E. (2016). Nuevas aproximaciones en el estudio de las propiedades psicométricas del STAI. Actas Españolas De Psiquiatría, 44(3), 83-92.

Pappa, S., Ntella, V., Giannakas, T., Giannakoulis, V. G., Papoutsi, E., \& Katsaounou, P. (2020). Prevalence of depression, anxiety, and insomnia among healthcare workers during the COVID-19 pandemic: A systematic review and meta-analysis. Brain, Behavior, and Immunity, 88, 901-907. https://doi.org/10. 1016/j.bbi.2020.05.026

Pinheiro, J., Bates, D., DebRoy, S., Sarkar, D., \& R Core Team (2020). nlme: Linear and nonlinear mixed effects models. R package version 3.1-148. CRAN.R project, 2020 https://CRAN.R-project.org/packa ge $=$ nlme

Programa de las Naciones Unidas para el Desarrollo [PNUD]. (2018). Aportes para el desarrollo humano en Argentina 2018: Género en el sector salud: feminización y brechas laborales [Contributions to human development in Argentina 2018: Gender in the health sector: feminization and labor gaps]. PNUD. https://www.argentina.gob.ar/sites/default/files/pnud_informedegenero_2018.04.04.pdf. Accessed 4 Oct 2021

R Core Team (2020). R: A language and environment for statistical computing. R Foundation for Statistical Computing. https://www.R-project.org/

Rettie, H., \& Daniels, J. (2020). Coping and tolerance of uncertainty: Predictors and mediators of mental health during the COVID-19 pandemic. The American Psychologist, [Epub ahead of print] 3 Aug 2020. https://doi.org/10.1037/amp0000710

Serrano-Ripoll, M. J., Meneses-Echavez, J. F., Ricci-Cabello, I., Fraile-Navarro, D., Fiol-deRoque, M. A., Pastor-Moreno, G., Castro, A., Ruiz-Pérez, I., Zamanillo Campos, R., \& Gonçalves-Bradley, D. C. (2020). Impact of viral epidemic outbreaks on mental health of healthcare workers: A rapid systematic review and meta-analysis. Journal of Affective Disorders, 277, 347-357. https://doi.org/10.1016/j.jad. 2020.08.034

Shechter, A., Diaz, F., Moise, N., Anstey, D. E., Ye, S., Agarwal, S., Birk, J. L., Brodie, D., Cannone, D. E., Chang, B., Claassen, J., Cornelius, T., Derby, L., Dong, M., Givens, R. C., Hochman, B., Homma, S., Kronish, I. M., Lee, S., Manzano, W., ... Abdalla, M. (2020). Psychological distress, coping behaviors, and preferences for support among New York healthcare workers during the COVID-19 pandemic. General Hospital Psychiatry, 66, 1-8. https://doi.org/10.1016/j.genhosppsych.2020.06.007

Spielberger, Ch., Gorsuch, R. L., \& Lushene, R. E. (1983). Manual for the State-Trait Anxiety Inventory. Consulting Psychologists Press.

Spoorthy, M. S., Pratapa, S. K., \& Mahant, S. (2020). Mental health problems faced by healthcare workers due to the COVID-19 pandemic-A review. Asian Journal of Psychiatry, 51, 102119. https://doi.org/10. 1016/j.ajp.2020.102119

Su, T. P., Lien, T. C., Yang, C. Y., Su, Y. L., Wang, J. H., Tsai, S. L., \& Yin, J. C. (2007). Prevalence of psychiatric morbidity and psychological adaptation of the nurses in a structured SARS caring unit during outbreak: A prospective and periodic assessment study in Taiwan. Journal of Psychiatric Research, 41(1-2), 119-130. https://doi.org/10.1016/j.jpsychires.2005.12.006 
Walton, M., Murray, E., \& Christian, M. D. (2020). Mental health care for medical staff and affiliated healthcare workers during the COVID-19 pandemic. European Heart Journal. Acute Cardiovascular Care, 9(3), 241-247.

Publisher's Note Springer Nature remains neutral with regard to jurisdictional claims in published maps and institutional affiliations. 